\title{
On the error term in Weyl's law for the Heisenberg manifolds(II)
}

\author{
ZHAI Wenguang
}

Science in China series A: Mathematics, Vol. 52(2009),857-874

\begin{abstract}
In this paper we study the mean square of the error term in the Weyl's law of an irrational $(2 l+1)$-dimensional Heisenberg manifold . An asymptotic formula is established.
\end{abstract}

\section{Introduction}

Let $(M, g)$ be a closed $n$-dimensional Riemannian manifold with metric $g$ and Laplace-Beltrami operator $\Delta$. Let $N(t)$ denote its spectral counting function, which is defined as the number of the eigenvalues of $\Delta$ not exceeding $t$. Hörmander [1] proved that the Weyl's law

$$
N(t)=\frac{\operatorname{vol}\left(B_{n}\right) \operatorname{vol}(M)}{(2 \pi)^{n}} t^{n / 2}+O\left(t^{(n-1) / 2}\right)
$$

holds, where $\operatorname{vol}\left(B_{n}\right)$ is the volume of the $n$-dimensional unit ball.

Let

$$
R(t):=N(t)-\frac{\operatorname{vol}\left(B_{n}\right) \operatorname{vol}(M)}{(2 \pi)^{n}} t^{n / 2} .
$$

Hörmander's estimate (1.1) in general is sharp, as the well-known example of the sphere $S^{n}$ with its canonical metric shows [11]. However, it is a very difficult problem to determine the optimal bound of $R(t)$ in any given manifold, which depends on the

2000 Mathematics Subject Classification: 11N37, 35P20, 58J50.

Key Words: Heisenberg manifold, Weyl's law, error term, mean square.

This work is supported by National Natural Science Foundation of China(Grant No. 10771127). 
properties of the associated geodesic flow. Many improvements have been obtained for certain types of manifolds, see [1, 2, 3, 4, 6, 8, 12, 13, 16, 21, 27].

\subsection{The Weyl's law for $\mathbb{T}^{2}$ : the Gauss circle problem}

The simplest compact manifold with integrable geodesic flow is the 2-torus $\mathbb{T}^{2}=\mathbb{R}^{2} / \mathbb{Z}^{2}$. The exponential functions $e(m x+n y)(m, n \in \mathbb{Z})$ form a basis of eigenfunctions of the Laplace operator $\Delta=\partial_{x}^{2}+\partial_{y}^{2}$, which acts on functions on $\mathbb{T}^{2}$. The corresponding eigenvalues are $4 \pi^{2}\left(m^{2}+n^{2}\right), m, n \in \mathbb{Z}$. The spectral counting function

$$
N_{I}(t)=\left\{\lambda_{j} \in \operatorname{Spec}(\Delta): \lambda_{j} \leq t\right\}
$$

is equal to the number of lattice points of $\mathbb{Z}^{2}$ inside a circle of radius $\sqrt{t} / 2 \pi$. The well-known Gauss circle problem is to study the properties of the error term of the function $N_{I}(t)$.

In this case, the formula (1.1) becomes

$$
N_{I}(t)=\frac{t}{4 \pi}+O\left(t^{1 / 2}\right)
$$

which is the classical result of Gauss. Let $R_{I}(t)$ denote the error term in (1.2). Many authors improved the upper bound estimate of $R_{I}(t)$. The latest result is due to Huxley[12], which reads

$$
R_{I}(t) \ll t^{131 / 416} \log ^{26947 / 8320} t .
$$

Hardy 9] conjectured that

$$
R_{I}(t) \ll t^{1 / 4+\varepsilon},
$$

which is supported by the asymptotic formula

$$
\int_{1}^{T}\left|R_{I}(t)\right|^{2} d t=\frac{1}{6 \pi^{3}} \sum_{n=1}^{\infty} \frac{r^{2}(n)}{n^{3 / 2}} T^{3 / 2}+O\left(T \log ^{2} T\right)
$$

proved in [14], where $r(n)$ denotes the number of ways $n$ can be written as a sum of two squares.

Tsang 24 first proved that the asymptotic formula

$$
\int_{1}^{T} R_{I}^{k}(t) d t=c_{k} T^{1+k / 4}+O\left(T^{1+k / 4-\delta_{k}+\varepsilon}\right)
$$

holds for $k=3$ and $k=4$ with $\delta_{3}=1 / 14$ and $\delta_{4}=1 / 23$, where $c_{k}(k \geq 3)$ and $\delta_{k}>0(k \geq 3)$ are explicit constants.

In [28], the author proved that (1.5) holds for any integer $3 \leq k \leq 9$. When $k=4$, in [29] the author proved that we can take $\delta_{4}=3 / 28$ in (1.5). 


\subsection{The Weyl's law for $(2 l+1)$-dimensional rational Heisen- berg manifold}

Let $l \geq 1$ be a fixed integer and $\left(H_{l} / \Gamma, g\right)$ be a $(2 l+1)$-dimensional Heisenberg manifold with a metric $g$. When $l=1$, in [21] Petridis and Toth proved that $R(t)=O\left(t^{5 / 6} \log t\right)$ for a special metric. Later in [4] this bound was improved to $O\left(t^{119 / 146+\varepsilon}\right)$ for all left-invariant Heisenberg metrics. For $l>1$ Khosravi and Petridis[16] proved that $R(t)=O\left(t^{l-7 / 41}\right)$ holds for rational Heisenberg manifolds. Both in [4] and [16, they first established a $\psi$-expression of $R(t)$ and then used the van der Corput method of exponential sums . If substituting Huxley's result of [12] into the arguments of [4] and [16], we can get that the estimate

$$
R(t)=O\left(t^{l-77 / 416}(\log t)^{26947 / 8320}\right)
$$

holds for all rational $(2 l+1)$-dimensional Heisenberg manifolds, which corresponds to Huxley's result (1.3).

It was conjectured that for rational Heisenberg manifolds, the pointwise estimate

$$
R(t) \ll t^{l-1 / 4+\varepsilon}
$$

holds, which was proposed in Petridis and Toth [21] for the case $l=1$ and in Khosravi and Petridis[16] for the case $l>1$. As an evidence of this conjecture, Petridis and Toth proved the following $L^{2}$ result for $H_{1}$

$$
\int_{I^{3}}\left|N(t ; u)-\frac{1}{6 \pi^{2}} \operatorname{vol}(M(u)) t^{3 / 2}\right|^{2} d u \leq C_{\delta} t^{3 / 2+\delta},
$$

where $I=[1-\varepsilon, 1+\varepsilon]$. They also proved

$$
\frac{1}{T} \int_{T}^{2 T}\left|N(t)-\frac{1}{6 \pi^{2}} \operatorname{vol}(M) t^{3 / 2}\right| d t \gg T^{3 / 4}
$$

Let $M=\left(H_{l} / \Gamma, g_{l}\right)$ be a $(2 l+1)$-dimensional Heisenberg manifold with the metric

$$
g_{l}:=\left(\begin{array}{ll}
I_{2 l \times 2 l} & 0 \\
0 & 2 \pi
\end{array}\right),
$$

where $I_{2 l \times 2 l}$ is the identity matrix.

M. Khosravi and John A. Toth[17] proved that

$$
\int_{1}^{T} R^{2}(t) d t=C_{2, l} T^{2 l+1 / 2}+O\left(T^{2 l+1 / 4+\varepsilon}\right),
$$


where $C_{2, l}$ is an explicit constant .

M. Khosravi [15] proved that the asymptotic formula

$$
\int_{1}^{T} R^{3}(t) d t=C_{3, l} T^{3 l+1 / 4}+O\left(T^{3 l+3 / 14+\varepsilon}\right)
$$

is true for some explicit constant $C_{3, l}$.

Recently, the author [30] proved that the asymptotic formula

$$
\int_{1}^{T} R^{k}(t) d t=C_{k, l} T^{k(l-1 / 4)+1}+O\left(T^{k(l-1 / 4)+1-\eta_{k}+\varepsilon}\right)
$$

holds for any $3 \leq k \leq 9$, where $C_{k, l}$ and $\eta_{k}>0$ are explicit constants. Especially (1.10) holds for $k=3$ with $\eta_{3}=1 / 4$ and for $k=4$ with $\eta_{4}=3 / 28$.

The moments problem of $R(t)$ becomes very difficult for the irrational Heisenberg manifolds even when we study only the mean square case. For the definition of rationality of Heisenberg manifolds, see[16. The aim of this paper is to study the mean square of the error term in the Weyl's law for the $(2 l+1)$-dimensional irrational Heisenbergs.

The plan of this paper is as follows. In Section 2 we shall state our main results. In Section 3 we state some background of the Heisenberg manifolds and give a $\psi$ expression of $R(t)$. In Section 4 are some preliminary Lemmas. We shall prove our theorem in Section 5.

Notations. For a real number $t$, let $[t]$ denote the integer part of $t,\{t\}=t-[t]$, $\psi(t)=\{t\}-1 / 2,\|t\|=\min (\{t\}, 1-\{t\}), e(t)=e^{2 \pi i t} . \varepsilon$ always denotes a sufficiently small positive constant. $\mathbb{C}, \mathbb{R}, \mathbb{Z}, \mathbb{N}$ denote the set of complex numbers, the set of real numbers, the set of integers, the set of positive integers, respectively. $n \sim M$ means that $N<n \leq 2 N$ and $n \asymp N$ means $c_{1} N \leq n \leq c_{2} N$ for some positive constants $0<c_{1}<c_{2} . S C(\Sigma)$ denotes the summation condition of the sum $\Sigma$. Throughout this paper, $\mathcal{L}$ always denotes $\log T$.

\section{Main results}

From now on, we always suppose that $R(t)$ denote the error term in the Weyl's law for the $(2 l+1)$-dimensional Heisenberg manifold with the irrational metric

$$
g_{l}(\theta):=\left(\begin{array}{ll}
I_{2 l \times 2 l} & 0 \\
0 & 2 \pi / \theta
\end{array}\right)
$$

where $\theta>0$ is an irrational number. 
The mean square of $R(t)$ is closely related to the approximation type of $\theta$. We recall a few facts from the theory of Diophantine approximation: by the approximation type $\gamma(\alpha)$ of an irrational real number $\alpha$ we denote the infimum of all reals $r$ for which there exists a constant $c(r, \alpha, \epsilon)$ such that the inequality

$$
|\alpha-p / q| \geq c(r, \alpha, \varepsilon) q^{-r-1-\varepsilon},
$$

for any $p \in \mathbb{Z}$ and any $q \in \mathbb{N}$. Obviously $\gamma(\alpha) \geq 1$ for all irrationals. By Roth's theorem [22], if $\alpha$ is an algebraic irrational, then $\gamma(\alpha)=1$. Furthermore, $\gamma(\alpha)=1$ for almost all irrationals(see Khinchin[18]).

Theorem 1. Suppose $\theta>0$ is an irrational number of finite type $\gamma$. Then we have

$$
\int_{1}^{T} R^{2}(x) d x=\frac{2^{9 / 2-4 l} \mathcal{C}_{l, \theta}}{(4 l+1)(l-1) !^{2} \pi^{2 l+3 / 2}} T^{2 l+1 / 2}+O\left(T^{2 l+\frac{4 \gamma+1}{8 \gamma+4}+\varepsilon}\right),
$$

where

$$
\mathcal{C}_{l, \theta}:=\sum_{h=1}^{\infty} \sum_{r>\theta h} \frac{\left(1-\frac{\theta h}{2 r-\theta h}\right)^{2 l-2}}{h^{1 / 2}(2 r-\theta h)^{5 / 2}} .
$$

Corollary. If $\theta$ is an algebraic irrational, then we have

$$
\int_{1}^{T} R^{2}(x) d x=\frac{2^{9 / 2-4 l} \mathcal{C}_{l, \theta}}{(4 l+1)(l-1) !^{2} \pi^{2 l+3 / 2}} T^{2 l+1 / 2}+O\left(T^{2 l+\frac{5}{12}+\varepsilon}\right) .
$$

Furthermore (2.3) holds for almost all irrational number $\theta>0$.

For the 3-dimensional case, we have the following more general Theorem 2 . We omit its proof since it is almost the same as that of Theorem 1.

Theorem 2. Let $R(t)$ denote the error term in the Weyl's law for the 3dimensional Heisenberg manifold with the metric

$$
g:=\left(\begin{array}{ccc}
h & & 0 \\
& 0 & g_{3}
\end{array}\right)
$$

where $g_{3}>0, h=\left(h_{i j}\right)(i, j=1,2)$ with $h_{11} h_{22}-h_{12}^{2}>0$. Suppose that $\pm i d^{2}(d>0)$ are the eigenvalues of the matrix $h^{-1} J$, where $J$ is the standard symplectic $2 \times 2$ matrix. Let $\theta=2 \pi / g_{3} d^{2}$.

If $\theta$ is an irrational number of finite approximation type $\gamma$, then we have

$$
\int_{1}^{T} R^{2}(x) d x=\frac{2^{1 / 2} \mathcal{C}_{1, \theta}}{5 d^{3} \pi^{7 / 2}} T^{5 / 2}+O\left(T^{\frac{20 \gamma+9}{8 \gamma+4}+\varepsilon}\right) .
$$

Remark. If the value $\theta$ in Theorem 2 is a rational number, then the error term $O\left(T^{\frac{20 \gamma+9}{8 \gamma+4}+\varepsilon}\right)$ in $(2.4)$ can be replaced by $O\left(T^{9 / 4+\varepsilon}\right)$. 


\section{Background of Heisenberg manifolds and the $\psi$-expression of $R(t)$}

In this section, we first review some background of the Heisenberg manifolds. The reader can see [5], [7], [23] for more details. Finally, we give an $\psi$-expression of $R(t)$.

\subsection{Heisenberg manifolds}

Suppose $x \in \mathbb{R}^{l}$ is a row vector and $y \in \mathbb{R}^{l}$ is a column vector. Define

$$
\gamma(x, y, t)=\left(\begin{array}{ccc}
1 & x & t \\
0 & I_{l} & y \\
0 & 0 & 1
\end{array}\right), \quad X(x, y, t)=\left(\begin{array}{ccc}
0 & x & t \\
0 & 0 & y \\
0 & 0 & 0
\end{array}\right)
$$

The $(2 l+1)$-dimensional Heisenberg group $H_{l}$ is defined by

$$
H_{l}=\left\{\gamma(x, y, t): x, y \in \mathbb{R}^{l}, t \in \mathbb{R}\right\}
$$

its Lie algebra is

$$
\mathfrak{H}_{l}=\left\{X(x, y, t): x, y \in \mathbb{R}^{l}, t \in \mathbb{R}\right\} .
$$

We say $\Gamma$ is uniform discrete subgroup of $H_{l}$ if $H_{l} / \Gamma$ is compact. A $(2 l+1)$ dimensional Heisenberg manifold is a pair $\left(H_{l} / \Gamma, g\right)$ for which $\Gamma$ is a uniform discrete subgroup of $H_{l}$ and $g$ is a left $H_{l}$-invariant metric.

For every $r$-tuple $\left(r_{1}, r_{2}, \cdots, r_{l}\right) \in \mathbb{N}^{l}$ such that $r_{j} \mid r_{j+1}(j=1,2, \cdots, l-1)$, let $r \mathbb{Z}^{l}$ denote the $l$-tuples $x=\left(x_{1}, x_{2}, \cdots, x_{l}\right)$ with $x_{j} \in r_{j} \mathbb{Z}$. Define

$$
\Gamma_{r}=\left\{\gamma(x, y, t): x \in r \mathbb{Z}^{l}, y \in r \mathbb{Z}^{l}, t \in \mathbb{Z}\right\} .
$$

It is clear that $\Gamma_{r}$ is a uniform discrete subgroup of $H_{l}$. According to Theorem 2.4 of [7], the subgroup $\Gamma_{r}$ classifies all the uniform discrete subgroups of $H_{l}$ up to automorphisms. Thus (see [7], Corollary 2.5) given any Riemannian Heisenberg manifold $M=\left(H_{l} / \Gamma, g\right)$, there exists a unique $l$-tuple $r$ as before and a left-invariant metric $\tilde{g}$ on $H_{l}$ such that $M$ is isometric to $\left(H_{l} / \Gamma, \tilde{g}\right)$. So (see [7], 2.6(b)) we can replace the metric $g$ by $\phi^{*} g$, where $\phi$ is an inner automorphism such that the direct sum split of the Lie algebra $\mathfrak{H}_{l}=\mathbb{R}^{2 l} \oplus \mathfrak{Z}$ is orthogonal. Here $\mathfrak{Z}$ is the center of the Lie algebra and

$$
\mathbb{R}^{2 l}=\left\{\left(\begin{array}{lll}
0 & x & 0 \\
0 & 0 & y \\
0 & 0 & 0
\end{array}\right): x, y \in \mathbb{R}^{l}\right\}
$$


With respect to this orthogonal split of $H_{l}$ the metric $g$ has the form

$$
\left(\begin{array}{ll}
h & 0 \\
0 & g_{2 l+1}
\end{array}\right)
$$

where $h$ is a positive-definite $2 l \times 2 l$ matrix and $g_{2 l+1}>0$ is a real number.

The volume of the Heisenberg manifold is given by

$$
\operatorname{vol}\left(H_{l} / \Gamma, g\right)=\left|\Gamma_{r}\right| \sqrt{\operatorname{det}(g)}
$$

with $\left|\Gamma_{r}\right|=r_{1} r_{2} \cdots r_{l}$ for $r=\left(r_{1}, r_{2}, \cdots, r_{l}\right)$.

\subsection{The spectrum of Heisenberg manifolds and the $\psi$-expression of $R(t)$}

Let $\Sigma$ be the spectrum of the Laplacian on $M=\left(H_{l} / \Gamma, g_{l}(\theta)\right)$, where the eigenvalues are counted with multiplicities. According to [7](P. 258), $\Sigma$ can be divided into two parts $\Sigma_{I}$ and $\Sigma_{I I}$, where $\Sigma_{I}$ is the spectrum of $2 l$-dimensional torus and $\Sigma_{I I}$ contains all eigenvalues of the form

$$
\frac{4 \pi^{2} m^{2}}{g_{2 l+1}}+\sum_{j=1}^{l} 2 \pi m\left(2 n_{j}+1\right), \quad m \in \mathbb{N}, n_{j} \in \mathbb{N} \cup\{0\},
$$

each eigenvalue counted with the multiplicity $2 m^{l}$.

We have the following $\psi$-expression of $R(t)$.

Lemma 3.1. We have

$$
\begin{gathered}
R(2 \pi x)=-\frac{4}{2^{l}(l-1) !} \sum_{1 \leq m \leq \sqrt{\frac{x}{\theta}}} m\left(x-\theta m^{2}\right)^{l-1} \psi\left(\frac{x}{2 m}-\frac{\theta m}{2}-\frac{l}{2}\right) \\
+O\left(x^{l-1 / 2}\right) .
\end{gathered}
$$

In [30, the author proved Lemma 3.1 when $\theta=1$. However, the proof for the general case is almost the same. So we omit the details of the proof.

\section{Some preliminary Lemmas}

We need the following Lemmas. Lemma 4.1 is due to Vaaler[25]. Lemma 4.2 is wellknown; see for example, Heath-Brown[10. Lemma 4.3 is Theorem 2.2 of Min[20, see also Lemma 6 of Chapter 1 in [26]. A weaker version of Lemma 4.3 can be found 
in [19], which also suffices for our proof. Lemma 4.4 and Lemma 4.6 provide several estimates about the quantity

$$
\alpha\left(\theta ; h_{1}, h_{2}, n_{1}, n_{2}\right):=\sqrt{h_{1}\left(2 n_{1}-\theta h_{1}\right)}-\sqrt{h_{2}\left(2 n_{2}-\theta h_{2}\right)},
$$

which play an essential role in our proof.

Lemma 4.1. Let $H \geq 2$ be any real number. Then

$$
\psi(u)=\sum_{1 \leq|h| \leq H} a(h) e(h u)+O\left(\sum_{0 \leq|h| \leq H} b(h) e(h u)\right),
$$

where $a(h)$ and $b(h)$ are functions such that $a(h) \ll 1 /|h|, b(h) \ll 1 / H$.

Lemma 4.2. Let $H \geq 2$ be any real number. Then

$$
\psi(u)=-\sum_{1 \leq|h| \leq H} \frac{e(h u)}{2 \pi i h}+O\left(\min \left(1, \frac{1}{H\|u\|}\right)\right) .
$$

Lemma 4.3. Suppose $A_{1}, \cdots, A_{5}$ are absolute positive constants, $f(x)$ and $g(x)$ are algebraic functions in $[a, b]$ and

$$
\begin{aligned}
& \frac{A_{1}}{R} \leq\left|f^{\prime \prime}(x)\right| \leq \frac{A_{2}}{R}, \quad\left|f^{\prime \prime \prime}(x)\right| \leq \frac{A_{3}}{R U}, \quad U \geq 1, \\
& |g(x)| \leq A_{4} G, \quad\left|g^{\prime}(x)\right| \leq A_{5} G U_{1}^{-1}, \quad U_{1} \geq 1,
\end{aligned}
$$

$[\alpha, \beta]$ is the image of $[a, b]$ under the mapping $y=f^{\prime}(x)$, then

$$
\begin{aligned}
\sum_{a<n \leq b} g(n) e(f(n))= & e^{\pi i / 4} \sum_{\alpha<u \leq \beta} b_{u} \frac{g\left(n_{u}\right)}{\sqrt{f^{\prime \prime}\left(n_{u}\right)}} e\left(f\left(n_{u}\right)-u n_{u}\right) \\
& +O\left(G \log (\beta-\alpha+2)+G(b-a+R)\left(U^{-1}+U_{1}^{-1}\right)\right) \\
& +O\left(G \min \left[\sqrt{R}, \max \left(\frac{1}{<\alpha>}, \frac{1}{<\beta>}\right)\right]\right),
\end{aligned}
$$

where $n_{u}$ is the solution of $f^{\prime}(n)=u$,

$$
\begin{gathered}
<t>= \begin{cases}\|t\|, & \text { if } t \text { not an integer, } \\
\beta-\alpha, & \text { if } t \text { an integer, }\end{cases} \\
b_{u}= \begin{cases}1, & \text { if } \alpha<u<\beta, \text { or } \alpha, \beta \text { not integers }, \\
1 / 2, & \text { if } \alpha \text { or } \beta \text { are integers, }\end{cases} \\
\sqrt{f^{\prime \prime}}= \begin{cases}\sqrt{f^{\prime \prime},} & \text { if } f^{\prime \prime}>0, \\
i \sqrt{\left|f^{\prime \prime}\right|}, & \text { if } f^{\prime \prime}<0 .\end{cases}
\end{gathered}
$$


Lemma 4.4. Suppose $\theta>0$ is an irrational number, $H_{1} \geq 2, H_{2} \geq 2, N_{1} \geq$ $2, N_{2} \geq 2, \Delta>0$. Let $\mathcal{A}_{\theta}\left(H_{1}, H_{2}, N_{1}, N_{2} ; \Delta\right)$ denote the number of solutions of the inequality

$$
\left|\sqrt{h_{1}\left(2 n_{1}-\theta h_{1}\right)}-\sqrt{h_{2}\left(2 n_{2}-\theta h_{2}\right)}\right| \leq \Delta
$$

for which

$$
h_{1} \sim H_{1}, h_{2} \sim H_{2}, n_{1} \sim N_{1}, n_{2} \sim N_{2}, n_{1}>\theta h_{1}, n_{2}>\theta h_{2},
$$

then

$$
\begin{aligned}
\mathcal{A}_{\theta}\left(H_{1}, H_{2}, N_{1}, N_{2} ; \Delta\right) \ll & \Delta\left(H_{1} H_{2} N_{1} N_{2}\right)^{3 / 4} \\
& +\left(H_{1} H_{2} N_{1} N_{2}\right)^{1 / 2} \log ^{2} H_{1} H_{2} N_{1} N_{2} .
\end{aligned}
$$

Proof. If $\Delta \geq\left(H_{1} H_{2} N_{1} N_{2}\right)^{1 / 4} / 100$, then trivially we have

$$
\mathcal{A}_{\theta}\left(H_{1}, H_{2}, N_{1}, N_{2} ; \Delta\right) \ll H_{1} H_{2} N_{1} N_{2} \ll \Delta\left(H_{1} H_{2} N_{1} N_{2}\right)^{3 / 4} .
$$

Now suppose $\Delta<\left(H_{1} H_{2} N_{1} N_{2}\right)^{1 / 4} / 100$. In this case $H_{1} N_{1} \asymp H_{2} N_{2}$. Without loss of generality, suppose $H_{1} \leq H_{2}$, then $N_{1} \gg N_{2}$. It is easy to see that if (4.1) holds, then

$$
\begin{aligned}
\left|2\left(h_{1} n_{1}-h_{2} n_{2}\right)-\theta\left(h_{1}^{2}-h_{2}^{2}\right)\right| & \leq \Delta\left(\sqrt{h_{1} n_{1}}+\sqrt{h_{2} n_{2}}\right) \\
& \leq 2 \Delta\left(\sqrt{H_{1} N_{1}}+\sqrt{H_{2} N_{2}}\right) \\
& \leq C \Delta \sqrt[4]{H_{1} N_{1} H_{2} N_{2}}
\end{aligned}
$$

for some $C>0$. Thus we have

$$
\frac{h_{2} n_{2}}{h_{1}}+\frac{\theta\left(h_{1}^{2}-h_{2}^{2}\right)}{2 h_{1}}-\frac{C \Delta\left(H_{1} N_{1} H_{2} N_{2}\right)^{\frac{1}{4}}}{2 h_{1}}<n_{1} \leq \frac{h_{2} n_{2}}{h_{1}}+\frac{\theta\left(h_{1}^{2}-h_{2}^{2}\right)}{2 h_{1}}+\frac{C \Delta\left(H_{1} N_{1} H_{2} N_{2}\right)^{\frac{1}{4}}}{2 h_{1}},
$$

which implies that

$$
\begin{aligned}
& \mathcal{A}_{\theta}\left(H_{1}, H_{2}, N_{1}, N_{2} ; \Delta\right) \\
& \ll \sum_{h_{1} \sim H_{1}} \sum_{h_{2} \sim H_{2}} \sum_{n_{2} \sim N_{2}}\left[\frac{h_{2} n_{2}}{h_{1}}+\frac{\theta\left(h_{1}^{2}-h_{2}^{2}\right)}{2 h_{1}}+\frac{C \Delta\left(H_{1} N_{1} H_{2} N_{2}\right)^{\frac{1}{4}}}{2 h_{1}}\right] \\
& \quad-\sum_{h_{1} \sim H_{1}} \sum_{h_{2} \sim H_{2}} \sum_{n_{2} \sim N_{2}}\left[\frac{h_{2} n_{2}}{h_{1}}+\frac{\theta\left(h_{1}^{2}-h_{2}^{2}\right)}{2 h_{1}}-\frac{C \Delta\left(H_{1} N_{1} H_{2} N_{2}\right)^{\frac{1}{4}}}{2 h_{1}}\right] \\
& =\Sigma_{1}+\Sigma_{2}-\Sigma_{3},
\end{aligned}
$$


where

$$
\begin{aligned}
\Sigma_{1} & =\sum_{h_{1} \sim H_{1}} \sum_{h_{2} \sim H_{2}} \sum_{n_{2} \sim N_{2}} \frac{C \Delta\left(H_{1} N_{1} H_{2} N_{2}\right)^{\frac{1}{4}}}{h_{1}}, \\
\Sigma_{2} & =\sum_{h_{1} \sim H_{1}} \sum_{h_{2} \sim H_{2}} \sum_{n_{2} \sim N_{2}} \psi\left(\frac{h_{2} n_{2}}{h_{1}}+\frac{\theta\left(h_{1}^{2}-h_{2}^{2}\right)}{2 h_{1}}-\frac{C \Delta\left(H_{1} N_{1} H_{2} N_{2}\right)^{\frac{1}{4}}}{2 h_{1}}\right), \\
\Sigma_{3} & =\sum_{h_{1} \sim H_{1}} \sum_{h_{2} \sim H_{2}} \sum_{n_{2} \sim N_{2}} \psi\left(\frac{h_{2} n_{2}}{h_{1}}+\frac{\theta\left(h_{1}^{2}-h_{2}^{2}\right)}{2 h_{1}}+\frac{C \Delta\left(H_{1} N_{1} H_{2} N_{2}\right)^{\frac{1}{4}}}{2 h_{1}}\right) .
\end{aligned}
$$

Obviously we have

$$
\Sigma_{1} \ll \Delta\left(H_{1} N_{1} H_{2} N_{2}\right)^{\frac{1}{4}} H_{2} N_{2} \ll \Delta\left(H_{1} N_{1} H_{2} N_{2}\right)^{\frac{3}{4}}
$$

if noting $H_{1} N_{1} \asymp H_{2} N_{2}$.

Now we estimate $\Sigma_{2}$. Taking $H=H_{1}$ in Lemma 4.1 we get

$$
\begin{aligned}
\Sigma_{2}= & \sum_{h_{1} \sim H_{1}} \sum_{h_{2} \sim H_{2}} \sum_{1 \leq|h| \leq H_{1}} a^{*}\left(h, h_{1}, h_{2}\right) \sum_{n_{2} \sim N_{2}} e\left(\frac{h h_{2} n_{2}}{h_{1}}\right) \\
& +O\left(\sum_{h_{1} \sim H_{1}} \sum_{h_{2} \sim H_{2}} \sum_{0 \leq|h| \leq H_{1}} b^{*}\left(h, h_{1}, h_{2}\right) \sum_{n_{2} \sim N_{2}} e\left(\frac{h h_{2} n_{2}}{h_{1}}\right)\right) \\
& \ll H_{2} N_{2} \log H_{1}+\Sigma_{4},
\end{aligned}
$$

where

$$
\begin{aligned}
\Sigma_{4} & =\sum_{h_{1} \sim H_{1}} \sum_{h_{2} \sim H_{2}} \sum_{1 \leq h \leq H_{1}} \frac{1}{h}\left|\sum_{n_{2} \sim N_{2}} e\left(\frac{h h_{2} n_{2}}{h_{1}}\right)\right|, \\
a^{*}\left(h, h_{1}, h_{2}\right) & =a(h) e\left(\frac{\theta\left(h_{1}^{2}-h_{2}^{2}\right)}{2 h_{1}}-\frac{C \Delta\left(H_{1} N_{1} H_{2} N_{2}\right)^{\frac{1}{4}}}{2 h_{1}}\right) \ll 1 /|h|, \\
b^{*}\left(h, h_{1}, h_{2}\right) & =b(h) e\left(\frac{\theta\left(h_{1}^{2}-h_{2}^{2}\right)}{2 h_{1}}-\frac{C \Delta\left(H_{1} N_{1} H_{2} N_{2}\right)^{\frac{1}{4}}}{2 h_{1}}\right) \ll 1 / H_{1} .
\end{aligned}
$$

It is easy to see that

$$
\sum_{n_{2} \sim N_{2}} e\left(\frac{h h_{2} n_{2}}{h_{1}}\right) \ll \begin{cases}N_{2}, & h_{1} \mid h h_{2}, \\ \frac{1}{\left\|\frac{h h_{2}}{h_{1}}\right\|}, & h_{1} \backslash h h_{2} .\end{cases}
$$


Thus

$$
\begin{aligned}
\Sigma_{4} & \ll \Sigma_{5}+\Sigma_{6}, \\
\Sigma_{5} & \left.=N_{2} \sum_{h_{1} \sim H_{1}} \sum_{1 \leq h \leq H_{1}} \frac{1}{h} \sum_{\substack{h_{2} \sim H_{2} \\
h h_{2} \equiv 0(\bmod }} h_{1}\right) \\
\Sigma_{6} & =\sum_{h_{1} \sim H_{1}} \sum_{1 \leq h \leq H_{1}} \frac{1}{h} \sum_{\substack{h_{2} \sim H_{2} \\
h h_{2} \neq 0(\bmod }} \frac{1}{\left\|\frac{h h_{2}}{h_{1}}\right\|} .
\end{aligned}
$$

Writing $h=d h^{*}, h_{1}=d h_{1}^{*}, d=\left(h, h_{1}\right)$, we get

$$
\begin{aligned}
\Sigma_{5} & \ll N_{2} \sum_{d \ll H_{1}} \frac{1}{d} \sum_{\substack { h_{1}^{*} \sim \frac{H_{1}}{d} \\
\begin{subarray}{c}{h^{*} \leq H_{1} \\
\left(h^{*}, h_{1}^{*}\right)=1{ h _ { 1 } ^ { * } \sim \frac { H _ { 1 } } { d } \\
\begin{subarray} { c } { h ^ { * } \leq H _ { 1 } \\
( h ^ { * } , h _ { 1 } ^ { * } ) = 1 } }\end{subarray}} \frac{1}{h^{*}} \sum_{\substack{h_{2} \sim H_{2} \\
h_{2} \equiv 0\left(\bmod h_{1}^{*}\right)}} 1 \\
& \ll N_{2} \sum_{d \ll H_{1}} \frac{1}{d} \sum_{\substack { h_{1}^{*} \sim \frac{H_{1}}{d} \\
\begin{subarray}{c}{h^{*} \leq \frac{H_{1}}{d} \\
\left(h^{*}, h_{1}^{*}\right)=1{ h _ { 1 } ^ { * } \sim \frac { H _ { 1 } } { d } \\
\begin{subarray} { c } { h ^ { * } \leq \frac { H _ { 1 } } { d } \\
( h ^ { * } , h _ { 1 } ^ { * } ) = 1 } }\end{subarray}} \frac{1}{h^{*}} \frac{H_{2}}{h_{1}^{*}} \\
& \ll N_{2} H_{2} \sum_{d \ll H_{1}} \frac{1}{d} \sum_{h^{*} \leq \frac{H_{1}}{d}} \frac{1}{h^{*}} \\
& \ll N_{2} H_{2} \log ^{2} H_{1} \ll\left(H_{1} N_{1} H_{2} N_{2}\right)^{1 / 2} \log ^{2} H_{1}
\end{aligned}
$$

if noting that $H_{1} N_{1} \asymp H_{2} N_{2}$.

For $\Sigma_{6}$ we have

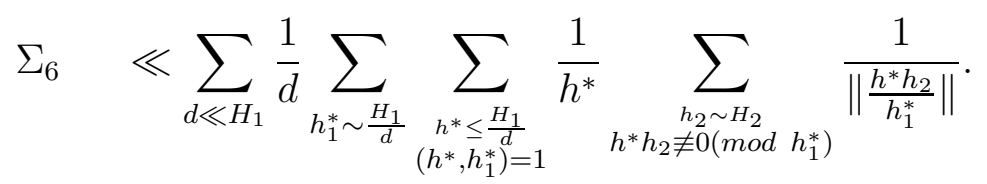

We need to bound the sum

$$
\sum_{\substack{h_{2} \sim H_{2} \\ h^{*} h_{2} \neq 0(\bmod }} \frac{1}{\left\|\frac{h^{*} h_{2}}{h_{1}^{*}}\right\|} .
$$

The condition $h^{*} h_{2} \not \equiv 0\left(\bmod h_{1}^{*}\right)$ implies that $h_{1}^{*} \geq 2$. Let $s=\left[H_{2} / h_{1}^{*}\right]$, then 
$s h_{1}^{*} \leq H_{2}<(s+1) h_{1}^{*}, 2 H_{2}<(2 s+2) h_{1}^{*}$. Thus

$$
\begin{aligned}
\sum_{\substack{h_{2} \sim H_{2} \\
h^{*} h_{2} \neq 0\left(\bmod h_{1}^{*}\right)}} \frac{1}{\left\|\frac{h^{*} h_{2}}{h_{1}^{*}}\right\|} & \ll \sum_{j=s}^{2 s+1} \sum_{\substack{j h_{1}^{*}<h_{2}<(j+1) h_{1}^{*} \\
h^{*} h_{2} \neq 0\left(\bmod h_{1}^{*}\right)}} \frac{1}{\left\|\frac{h^{*} h_{2}}{h_{1}^{*}}\right\|} \\
& \ll \sum_{j=s}^{2 s+1} \sum_{h_{1}^{*}=1}^{h_{1}^{*}-1} \frac{1}{\| \frac{h_{2}^{*} \|}{h_{1}^{*}}} \ll \sum_{j=s}^{2 s+1} \sum_{h_{2}^{*}=1}^{\left[h_{1}^{*} / 2\right]} \frac{1}{\| \frac{h_{2}^{*} \|}{h_{1}^{*}}} \\
& \ll \sum_{j=s}^{2 s+1} \sum_{h_{2}^{*}=1}^{\left[h_{1}^{*} / 2\right]} \frac{h_{1}^{*}}{h_{2}^{*}} \ll H_{2} \log H_{1} .
\end{aligned}
$$

Inserting this bound into (4.8) we get

$$
\Sigma_{6} \ll H_{1} H_{2} \log ^{2} H_{1} \ll\left(H_{1} N_{1} H_{2} N_{2}\right)^{1 / 2} \log ^{2} H_{1}
$$

if noting that $N_{1} \gg H_{1}$ and $N_{2} \gg H_{2}$.

From (4.4)-(4.9) we get

$$
\Sigma_{2} \ll \Delta\left(H_{1} H_{2} N_{1} N_{2}\right)^{3 / 4}+\left(H_{1} H_{2} N_{1} N_{2}\right)^{1 / 2} \log ^{2} H_{1} H_{2} N_{1} N_{2} .
$$

Similarly we have

$$
\Sigma_{3} \ll \Delta\left(H_{1} H_{2} N_{1} N_{2}\right)^{3 / 4}+\left(H_{1} H_{2} N_{1} N_{2}\right)^{1 / 2} \log ^{2} H_{1} H_{2} N_{1} N_{2} .
$$

Now Lemma 4.4 follows from (4.3), (4.10) and (4.11).

Lemma 4.5. Suppose $\theta>0$ is an irrational number of approximation type $\gamma \geq 1$. Then for any $q \in \mathbb{N} \bigcup(\mathbb{N}+1 / 2)$, we have

$$
\|q \theta\| \gg q^{-\gamma-\varepsilon}
$$

where the implied constant depending only on $\varepsilon$.

Proof. It follows easily from the definition of approximation type.

Lemma 4.6. Suppose $\theta>0$ is an irrational number of approximation type $\gamma \geq 1$ and that

$$
0<\left|\alpha\left(\theta ; h_{1}, h_{2}, n_{1}, n_{2}\right)\right|<\frac{1}{10} \beta^{1 / 4}\left(\theta ; h_{1}, h_{2}, n_{1}, n_{2}\right), n_{1}>\theta h_{1}, n_{2}>\theta h_{2},
$$

where

$$
\beta\left(\theta ; h_{1}, h_{2}, n_{1}, n_{2}\right):=h_{1}\left(2 n_{1}-\theta h_{1}\right) h_{2}\left(2 n_{2}-\theta h_{2}\right)
$$


If $h_{1}=h_{2}=h$, then

$$
\left|\alpha\left(\theta ; h, h, n_{1}, n_{2}\right)\right| \gg \frac{h^{1 / 2}}{\left(n_{1} n_{2}\right)^{1 / 4}} .
$$

If $h_{1} \neq h_{2}$, then

$$
\left|\alpha\left(\theta ; h_{1}, h_{2}, n_{1}, n_{2}\right)\right| \gg \frac{\left|h_{1} h_{2}\right|^{\frac{-\gamma-\varepsilon}{2}}}{\left(h_{1} h_{2} n_{1} n_{2}\right)^{1 / 4}} .
$$

Proof. The condition (4.12) implies that

$$
h_{1}\left(2 n_{1}-\theta h_{1}\right) \asymp h_{2}\left(2 n_{2}-\theta h_{2}\right) \asymp h_{1} n_{1} \asymp h_{2} n_{2} .
$$

So we have

$$
\begin{aligned}
\mid \alpha\left(\theta ; h_{1}, h_{2}, n_{1}, n_{2} \mid\right. & =\frac{\left|2\left(h_{1} n_{1}-h_{2} n_{2}\right)-\theta\left(h_{1}^{2}-h_{2}^{2}\right)\right|}{\sqrt{h_{1}\left(2 n_{1}-\theta h_{1}\right)}+\sqrt{h_{2}\left(2 n_{2}-\theta h_{2}\right)}} \\
& \gg \frac{\left|2\left(h_{1} n_{1}-h_{2} n_{2}\right)-\theta\left(h_{1}^{2}-h_{2}^{2}\right)\right|}{\left(h_{1} h_{2} n_{1} n_{2}\right)^{1 / 4}}
\end{aligned}
$$

If $h_{1}=h_{2}=h$, then (4.13) implies

$$
\mid \alpha\left(\theta ; h_{1}, h_{2}, n_{1}, n_{2} \mid \gg \frac{h\left|n_{1}-n_{2}\right|}{h^{1 / 2}\left(n_{1} n_{2}\right)^{1 / 4}} \gg \frac{h^{1 / 2}}{\left(n_{1} n_{2}\right)^{1 / 4}} .\right.
$$

If $h_{1} \neq h_{2}$, then from (4.13) and Lemma 4.5 we have

$$
\begin{aligned}
\mid \alpha\left(\theta ; h_{1}, h_{2}, n_{1}, n_{2} \mid\right. & \gg \frac{\left\|\theta\left(h_{1}^{2}-h_{2}^{2}\right)\right\|}{\left(h_{1} h_{2} n_{1} n_{2}\right)^{1 / 4}} \\
& \gg \frac{\left|h_{1}^{2}-h_{2}^{2}\right|^{-\gamma-\varepsilon}}{\left(h_{1} h_{2} r_{1} r_{2}\right)^{1 / 4}} \\
& \gg \frac{\left|h_{1}+h_{2}\right|^{-\gamma-\varepsilon}\left|h_{1}-h_{2}\right|^{-\gamma-\varepsilon}}{\left(h_{1} h_{2} r_{1} r_{2}\right)^{1 / 4}} \\
& \gg \frac{\left|h_{1}+h_{2}\right|^{-\gamma-\varepsilon}}{\left(h_{1} h_{2} r_{1} r_{2}\right)^{1 / 4}} \\
& \gg \frac{\left|h_{1} h_{2}\right|^{-\gamma-\varepsilon}}{\left(h_{1} h_{2} r_{1} r_{2}\right)^{1 / 4}},
\end{aligned}
$$

where in the last step we used the inequality $a^{2}+b^{2} \geq 2 a b$. 


\section{Proof of Theorem 1}

In this section we shall prove Theorem 1. It suffices for us to evaluate the integral $\int_{T}^{2 T} R^{2}(2 \pi x) d x$, where $T$ is a large real number.

Suppose $H:=T^{2}$. By Lemma 3.1 and Lemma 4.2 we have

$$
\begin{aligned}
& R_{1}(x ; H)=\frac{2^{1-l}}{(l-1) ! \pi i} \sum_{1 \leq|h| \leq H} \frac{e(-l h / 2)}{h} \sum_{m \leq \sqrt{\frac{x}{\theta}}} m\left(x-\theta m^{2}\right)^{l-1} e\left(h\left(\frac{x}{2 m}-\frac{\theta m}{2}\right)\right), \\
& R_{2}(x, H)=O\left(T^{l-1 / 2} G(x, H)+T^{l-1 / 2}\right), \\
& G(x, H)=\sum_{m \leq \sqrt{\frac{x}{\theta}}} \min \left(1, \frac{1}{H\left\|\frac{x}{2 m}-\frac{\theta m}{2}+\frac{l}{2}\right\|}\right) .
\end{aligned}
$$

\subsection{The mean-square of $R_{1}(x, H)$}

In this subsection we shall study The mean-square of $R_{1}(x, H)$. First we shall derive an Voronoï type formula for $R_{1}(x, H)$.

\subsubsection{Voronoï analogue formula for $R_{1}(x, H)$}

We have

$$
\begin{aligned}
& R_{1}(x, H)= \frac{2^{1-l}}{(l-1) ! \pi i} \sum_{1 \leq|h| \leq H} \frac{e(-l h / 2)}{h} \sum_{m \leq \sqrt{\frac{x}{\theta}}} m\left(x-\theta m^{2}\right)^{l-1} \\
& \times e\left(h\left(\frac{x}{2 m}-\frac{\theta m}{2}\right)\right) \\
&= \frac{2^{1-l}}{(l-1) ! \pi i} \sum_{j_{1}=0}^{l-1}(-\theta)^{j_{1}}\left(\begin{array}{c}
l-1 \\
j_{1}
\end{array}\right) \sum_{1 \leq|h| \leq H} \frac{e(-l h / 2)}{h} \sum_{m \leq \sqrt{\frac{x}{\theta}}} x^{l-1-j_{1}} \\
& \quad \times m^{2 j_{1}+1} e\left(h\left(\frac{x}{2 m}-\frac{\theta m}{2}\right)\right) \\
&=\frac{2^{1-l}}{(l-1) !} \sum_{j_{1}=0}^{l-1}(-\theta)^{j_{1}}\left(\begin{array}{c}
l-1 \\
j_{1}
\end{array}\right) F\left(x ; j_{1}\right)
\end{aligned}
$$


say, where

$$
F\left(x ; j_{1}\right):=\frac{1}{\pi i} \sum_{1 \leq|h| \leq H} \frac{e(-l h / 2)}{h} \sum_{m \leq \sqrt{\frac{x}{\theta}}} x^{l-1-j_{1}} m^{2 j_{1}+1} e\left(h\left(\frac{x}{2 m}-\frac{\theta m}{2}\right)\right) .
$$

Let $J=[(\mathcal{L}-\log \mathcal{L}) / 2 \log 2]$ we get

(5.3) $\quad F\left(x ; j_{1}\right)$

$$
\begin{aligned}
= & \frac{1}{\pi i} \sum_{-H \leq h \leq-1} \frac{e(-l h / 2)}{h} \sum_{j=0}^{J} \sum_{m \sim \sqrt{\frac{x}{\theta}} 2^{-j-1}} x^{l-1-j_{1}} m^{2 j_{1}+1} e\left(h\left(\frac{x}{2 m}-\frac{\theta m}{2}\right)\right) \\
& +\frac{1}{\pi i} \sum_{1 \leq h \leq H} \frac{e(-l h / 2)}{h} \sum_{j=0}^{J} \sum_{m \sim \sqrt{\frac{x}{\theta}} 2^{-j-1}} x^{l-1-j_{1}} m^{2 j_{1}+1} e\left(h\left(\frac{x}{2 m}-\frac{\theta m}{2}\right)\right)+O\left(x^{l-1} \mathcal{L}^{2}\right) \\
= & -\frac{1}{\pi i} \sum_{1 \leq h \leq H} \frac{e(l h / 2)}{h} \sum_{j=0}^{J} \sum_{m \sim \sqrt{\frac{x}{\theta}} 2^{-j-1}} x^{l-1-j_{1}} m^{2 j_{1}+1} e\left(-h\left(\frac{x}{2 m}-\frac{\theta m}{2}\right)\right) \\
& +\frac{1}{\pi i} \sum_{1 \leq h \leq H} \frac{e(-l h / 2)}{h} \sum_{j=0}^{J} \sum_{m \sim \sqrt{\frac{x}{\theta}} 2^{-j-1}} x^{l-1-j_{1}} m^{2 j_{1}+1} e\left(h\left(\frac{x}{2 m}-\frac{\theta m}{2}\right)\right)+O\left(x^{l-1} \mathcal{L}^{2}\right) \\
= & -\frac{\Sigma_{7}}{\pi i}+\frac{\Sigma_{7}}{\pi i}+O\left(x^{l-1} \mathcal{L}^{2}\right),
\end{aligned}
$$

where

$$
\Sigma_{7}=\sum_{1 \leq h \leq H} \frac{e(l h / 2)}{h} \sum_{j=0}^{J} \sum_{m \sim \sqrt{\frac{x}{\theta}} 2^{-j-1}} x^{l-1-j_{1}} m^{2 j_{1}+1} e\left(-h\left(\frac{x}{2 m}-\frac{\theta m}{2}\right)\right) .
$$

Let

$$
S\left(x ; h, j_{1}, j\right)=\sum_{m \sim \sqrt{\frac{x}{\theta}} 2^{-j-1}} x^{l-1-j_{1}} m^{2 j_{1}+1} e\left(-h\left(\frac{x}{2 m}-\frac{\theta m}{2}\right)\right) .
$$

By Lemma 4.3 we get

$$
\text { (5.4) } \begin{aligned}
S\left(x ; h, j_{1}, j\right) & =e^{-\frac{\pi i}{4}} \sum_{\beta(h, j)<r \leq \beta(h, j+1)} \frac{x^{l-1 / 4} h^{j_{1}+3 / 4}}{(2 r-\theta h)^{j_{1}+5 / 4}} e(-\sqrt{x h(2 r-\theta h)}) \\
& +O\left(x^{l-1 / 2} \mathcal{L}\right)+O\left(x^{l-1 / 2} \min \left(\frac{x^{\frac{1}{4}}}{h^{\frac{1}{2}} 2^{\frac{3 j}{2}}}, \frac{1}{\|\beta(h, j)\|}\right)\right) \\
& +O\left(x^{l-1 / 2} \min \left(\frac{x^{\frac{1}{4}}}{h^{\frac{1}{2}} 2^{\frac{3 j}{2}}}, \frac{1}{\|\beta(h, j+1)\|}\right)\right),
\end{aligned}
$$


where

$$
\beta(h, j):=\theta h\left(2^{2 j-1}+1 / 2\right) .
$$

Inserting (5.4) into $\Sigma_{7}$ we have

$$
\begin{gathered}
(5.6) \Sigma_{7}=e^{-\pi i / 4} \sum_{1 \leq h \leq H} \frac{e(l h / 2)}{h} \sum_{j=0}^{J} \sum_{\beta(h, j)<r \leq \beta(h, j+1)} \\
\frac{x^{l-1 / 4} h^{j_{1}+3 / 4}}{(2 r-\theta h)^{j_{1}+5 / 4}} e(-\sqrt{x h(2 r-\theta h)})+O(g(T, H)) \\
=\sum_{\substack{1 \leq h \leq H \\
+}} \frac{e(l h / 2)}{h} \sum_{\theta h<r \leq \theta h\left(2^{2 J+1}+1 / 2\right)} \frac{x^{l-1 / 4} h^{j_{1}+3 / 4}}{(2 r-\theta h)^{j_{1}+5 / 4}} e\left(-\sqrt{x h(2 r-\theta h)}-\frac{1}{8}\right) \\
+O(T, H)),
\end{gathered}
$$

where

$$
g(T, H):=T^{l-1 / 2} \mathcal{L}^{3}+T^{l-1 / 2} \sum_{1 \leq h \leq H} \frac{1}{h} \sum_{j=0}^{J} \min \left(\frac{T^{\frac{1}{4}}}{h^{\frac{1}{2}} 2^{\frac{3 j}{2}}}, \frac{1}{\|\beta(h, j)\|}\right) .
$$

By Lemma 4.5 we have $\|\beta(h, j)\| \gg\left(h 2^{2 j}\right)^{-\gamma-\varepsilon}$, which implies that

$$
\begin{aligned}
(5.7) g(T, H) & \ll T^{l-1 / 2} \mathcal{L}^{3}+T^{l-1 / 2} \sum_{1 \leq h \leq H} \frac{1}{h} \sum_{j=0}^{J} \min \left(\frac{T^{\frac{1}{4}}}{h^{\frac{1}{2}} 2^{\frac{3 j}{2}}},\left(h 2^{2 j}\right)^{\gamma+\varepsilon}\right) \\
& \ll T^{l-1 / 2} \mathcal{L}^{3}+T^{l-1 / 2} \sum_{1 \leq h \leq H} \frac{1}{h} \sum_{j=0}^{J}\left(\frac{T^{\frac{1}{4}}}{h^{\frac{1}{2}} 2^{\frac{3 j}{2}}}\right)^{\frac{2 \gamma}{2 \gamma+1}}\left(\left(h 2^{2 j}\right)^{\gamma+\varepsilon}\right)^{\frac{1}{2 \gamma+1}} \\
& \ll T^{l-1 / 2+\frac{\gamma}{4 \gamma+2}+\varepsilon} .
\end{aligned}
$$


Inserting (5.4)-(5.7) into (5.3) we get

$$
\begin{aligned}
& F\left(x ; j_{1}\right)=\frac{1}{\pi i} \sum_{1 \leq h \leq H} \frac{e(l h / 2)}{h} \sum_{\theta h<r \leq \theta h\left(2^{2 J+1}+1 / 2\right)} \frac{x^{l-1 / 4} h^{j_{1}+3 / 4}}{(2 r-\theta h)^{j_{1}+5 / 4}} \\
& \times\left(e\left(\sqrt{x h(2 r-\theta h)}+\frac{1}{8}\right)-e\left(\sqrt{x h(2 r-\theta h)}-\frac{1}{8}\right)\right)+O\left(T^{l-1 / 2+\frac{\gamma}{4 \gamma+2}+\varepsilon}\right) \\
& =\frac{2 x^{l-1 / 4}}{\pi} \sum_{1 \leq h \leq H} \frac{e(l h / 2)}{h} \sum_{\theta h<r \leq \theta h\left(2^{2 J+1}+1 / 2\right)} \frac{h^{j_{1}+3 / 4}}{(2 r-\theta h)^{j_{1}+5 / 4}} \sin \left(2 \pi \sqrt{x h(2 r-\theta h)}+\frac{\pi}{4}\right) \\
& +O\left(T^{l-1 / 2+\frac{\gamma}{4 \gamma+2}+\varepsilon}\right)
\end{aligned}
$$

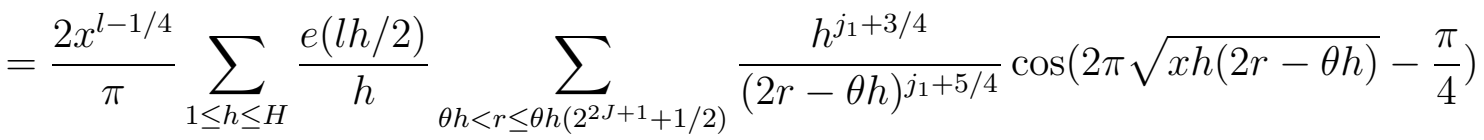

$$
\begin{aligned}
& +O\left(T^{l-1 / 2+\frac{\gamma}{4 \gamma+2}+\varepsilon}\right) \text {. }
\end{aligned}
$$

From (5.2) and (5.8) we get

$$
\begin{aligned}
& R_{1}(x, H)=\frac{2^{2-l} x^{l-1 / 4}}{(l-1) ! \pi} \sum_{1 \leq h \leq H} \sum_{\theta h<r \leq \theta h\left(2^{2 J+1}+1 / 2\right)} \cos \left(2 \pi \sqrt{x h(2 r-\theta h)}-\frac{\pi}{4}\right) \\
& \times \sum_{j_{1}=0}^{l-1}(-1)^{j_{1}}\left(\begin{array}{c}
l-1 \\
j_{1}
\end{array}\right) \frac{e(l h / 2) h^{j_{1}-1 / 4}}{(2 r-\theta h)^{j_{1}+5 / 4}}+O\left(T^{l-1 / 2+\frac{\gamma}{4 \gamma+2}+\varepsilon}\right) \\
& =R_{11}(x, H)+O\left(T^{l-1 / 2+\frac{\gamma}{4 \gamma+2}+\varepsilon}\right) \text {, }
\end{aligned}
$$

where

$$
\begin{aligned}
& R_{11}(x, H):= \frac{2^{2-l} x^{l-1 / 4}}{(l-1) ! \pi} \sum_{1 \leq h \leq H} \sum_{\theta h<r \leq \theta h\left(2^{2 J+1}+1 / 2\right)} u(h, r) \\
& \times \cos \left(2 \pi \sqrt{x h(2 r-\theta h)}-\frac{\pi}{4}\right), \\
& u(h, r):=\frac{e(l h / 2)}{h^{1 / 4}(2 r-\theta h)^{5 / 4}}\left(1-\frac{\theta h}{2 r-\theta h}\right)^{l-1} .
\end{aligned}
$$

\subsubsection{Mean square of $R_{11}(x, H)$}

Now we study the mean square of $R_{11}(x, H)$. By the elementary formula

$$
\cos u \cos v=\frac{1}{2}(\cos (u-v)+\cos (u+v))
$$


we have

$$
\begin{aligned}
R_{11}^{2}(x, H)= & \frac{2^{4-2 l} x^{2 l-\frac{1}{2}}}{\pi^{2}(l-1) \sum^{2}} \sum_{8} u\left(h_{1}, r_{1}\right) u\left(h_{2}, r_{2}\right) \\
& \times \prod_{j=1}^{2} \cos \left(2 \pi \sqrt{x h_{j}\left(2 r_{j}-\theta h_{j}\right)}-\frac{\pi}{4}\right) \\
= & S_{1}(x)+S_{2}(x)+S_{3}(x),
\end{aligned}
$$

where

$$
\begin{aligned}
& S_{1}(x)=\frac{2^{3-2 l} x^{2 l-\frac{1}{2}}}{\pi^{2}(l-1) !^{2}} \sum_{9} u\left(h_{1}, r_{1}\right) u\left(h_{2}, r_{2}\right), \\
& S_{2}(x)=\frac{2^{3-2 l} x^{2 l-\frac{1}{2}}}{\pi^{2}(l-1) !^{2}} \sum_{10} u\left(h_{1}, r_{1}\right) u\left(h_{2}, r_{2}\right) \cos \left(\alpha\left(\theta ; h_{1}, h_{2}, r_{1}, r_{2}\right)\right), \\
& S_{3}(x)=\frac{2^{3-2 l} x^{2 l-\frac{1}{2}}}{\pi^{2}(l-1) !^{2}} \sum_{8} u\left(h_{1}, r_{1}\right) u\left(h_{2}, r_{2}\right) \\
& \times \sin \left(2 \pi \sqrt{x h_{1}\left(2 r_{1}-\theta h_{1}\right)}+2 \pi \sqrt{x h_{2}\left(2 r_{2}-\theta h_{2}\right)}\right), \\
& \begin{array}{c}
S C\left(\Sigma_{8}\right): 1 \leq h_{j} \leq H, \theta h_{j}<r_{j} \leq \theta h_{j}\left(2^{2 J+1}+1 / 2\right) \quad(j=1,2), \\
S C\left(\Sigma_{9}\right): 1 \leq h_{j} \leq H, \theta h_{j}<r_{j} \leq \theta h_{j}\left(2^{2 J+1}+1 / 2\right) \quad(j=1,2), \\
\quad \alpha\left(\theta ; h_{1}, h_{2}, r_{1}, r_{2}\right)=0, \\
S C\left(\Sigma_{10}\right): 1 \leq h_{j} \leq H, \theta h_{j}<r_{j} \leq \theta h_{j}\left(2^{2 J+1}+1 / 2\right) \quad(j=1,2), \\
\alpha\left(\theta ; h_{1}, h_{2}, r_{1}, r_{2}\right) \neq 0 .
\end{array}
\end{aligned}
$$

We first consider the contribution of $S_{1}(x)$. Since $\theta$ is irrational, we see easily that $\alpha\left(\theta ; h_{1}, h_{2}, r_{1}, r_{2}\right)=0$ holds if and only if $h_{1}=h_{2}, r_{1}=r_{2}$. Thus we have

$$
\int_{T}^{2 T} S_{1}(x) d x=\frac{2^{3-2 l}}{\pi^{2}(l-1) !^{2}} \sum_{1 \leq h \leq H} \sum_{\theta h<r \leq \theta h\left(2^{2 J+1}+1 / 2\right)} u^{2}(h, r) \int_{T}^{2 T} x^{2 l-\frac{1}{2}} d x
$$


Recalling the definition of $u(h, r)$, we have

$$
\begin{aligned}
& \sum_{1 \leq h \leq H} \sum_{\theta h<r \leq \theta h\left(2^{2 J+1}+1 / 2\right)} u^{2}(h, r) \\
= & \sum_{1 \leq h \leq H} \sum_{\theta h<r} \frac{\left(1-\frac{\theta h}{2 r-\theta h}\right)^{2 l-2}}{h^{1 / 2}(2 r-\theta h)^{5 / 2}}+O\left(\sum_{1 \leq h \leq H} \sum_{r>\theta h 2^{2 J+1}} h^{-1 / 2} r^{-5 / 2}\right) \\
= & \sum_{h=1}^{\infty} \sum_{r>\theta h} \frac{\left(1-\frac{\theta h}{2 r-\theta h}\right)^{2 l-2}}{h^{1 / 2}(2 r-\theta h)^{5 / 2}}+O\left(T^{-3 / 2}+H^{-2}\right) \\
= & \mathcal{C}_{l, \theta}+O\left(T^{-3 / 2}\right),
\end{aligned}
$$

which combining (5.12) gives

$$
\int_{T}^{2 T} S_{1}(x) d x=\frac{2^{3-2 l} \mathcal{C}_{l, \theta}}{\pi^{2}(l-1) !^{2}} \int_{T}^{2 T} x^{2 l-\frac{1}{2}} d x+O\left(T^{2 l-1}\right) .
$$

For the contribution of $S_{3}(x)$, by the first derivative test we get

$$
\begin{aligned}
& \int_{T}^{2 T} S_{3}(x) d x \ll T^{2 l} \sum_{8} \frac{\left(\sqrt{h_{1}\left(2 r_{1}-\theta h_{1}\right)}+\sqrt{h_{2}\left(2 r_{2}-\theta h_{2}\right)}\right)^{-1}}{\left(h_{1} h_{2}\right)^{\frac{1}{4}}\left(2 r_{1}-\theta h_{1}\right)^{\frac{5}{4}}\left(2 r_{2}-\theta h_{2}\right)^{\frac{5}{4}}} \\
\ll & T^{2 l} \sum_{8} \frac{\left(\sqrt{h_{1}\left(2 r_{1}-\theta h_{1}\right)} \sqrt{h_{2}\left(2 r_{2}-\theta h_{2}\right)}\right)^{-1 / 2}}{\left(h_{1} h_{2}\right)^{\frac{1}{4}}\left(2 r_{1}-\theta h_{1}\right)^{\frac{5}{4}}\left(2 r_{2}-\theta h_{2}\right)^{\frac{5}{4}}} \\
\ll & T^{2 l} \sum_{8} \frac{1}{\left(h_{1} h_{2}\right)^{\frac{1}{2}}\left(2 r_{1}-\theta h_{1}\right)^{\frac{3}{2}}\left(2 r_{2}-\theta h_{2}\right)^{\frac{3}{2}}} \\
\ll & T^{2 l}\left(\sum_{1 \leq h \leq H} h^{-1 / 2} \sum_{\theta h<r \leq \theta h\left(2^{2 J+1}+1 / 2\right)} r^{-3 / 2}\right)^{2} \\
\ll & T^{2 l} \mathcal{L}^{2}, \quad
\end{aligned}
$$

where in the second step we used the inequality $a^{2}+b^{2} \geq 2 a b$. 
Finally we consider the contribution of $S_{2}(x)$. By the first derivative test we get

$$
\begin{gathered}
\int_{T}^{2 T} S_{2}(x) d x \\
\ll T^{2 l-1 / 2} \sum_{10} \frac{1}{h_{1}^{1 / 4} h_{2}^{1 / 4} r_{1}^{5 / 4} r_{2}^{5 / 4}} \min \left(T, \frac{T^{1 / 2}}{\left|\alpha\left(\theta ; h_{1}, h_{2}, r_{1}, r_{2}\right)\right|}\right) \\
\ll T^{2 l} \sum_{11} \frac{1}{h_{1}^{1 / 4} h_{2}^{1 / 4} r_{1}^{5 / 4} r_{2}^{5 / 4}} \min \left(T^{1 / 2}, \frac{1}{\left|\alpha\left(\theta ; h_{1}, h_{2}, r_{1}, r_{2}\right)\right|}\right) \\
+T^{2 l} \sum_{12} \frac{1}{h^{1 / 2} r_{1}^{5 / 4} r_{2}^{5 / 4}} \min \left(T^{1 / 2}, \frac{1}{\left|\alpha\left(\theta ; h, h, r_{1}, r_{2}\right)\right|}\right) \\
+T^{2 l} \sum_{13} \frac{1}{h_{1}^{1 / 4} h_{2}^{1 / 4} r_{1}^{5 / 4} r_{2}^{5 / 4}} \min \left(T^{1 / 2}, \frac{1}{\left|\alpha\left(\theta ; h_{1}, h_{2}, r_{1}, r_{2}\right)\right|}\right)
\end{gathered}
$$

where

$$
\begin{gathered}
S C\left(\Sigma_{11}\right): 1 \leq h_{j} \leq H, \theta h_{j}<r_{j} \leq \theta h_{j}\left(2^{2 J+1}+1 / 2\right) \quad(j=1,2), \\
\left|\alpha\left(\theta ; h_{1}, h_{2}, r_{1}, r_{2}\right)\right| \geq \frac{1}{10} \beta^{1 / 4}\left(\theta ; h_{1}, h_{2}, r_{1}, r_{2}\right), \\
S C\left(\Sigma_{12}\right): 1 \leq h \leq H, \theta h<r_{j} \leq \theta h\left(2^{2 J+1}+1 / 2\right)(j=1,2), r_{1} \neq r_{2}, \\
\left|\alpha\left(\theta ; h, h, r_{1}, r_{2}\right)\right|<\frac{1}{10} \beta^{1 / 4}\left(\theta ; h, h, r_{1}, r_{2}\right), \\
S C\left(\Sigma_{13}\right): 1 \leq h_{j} \leq H, \theta h_{j}<r_{j} \leq \theta h_{j}\left(2^{2 J+1}+1 / 2\right) \quad(j=1,2), h_{1} \neq h_{2}, \\
\left|\alpha\left(\theta ; h_{1}, h_{2}, r_{1}, r_{2}\right)\right|<\frac{1}{10} \beta^{1 / 4}\left(\theta ; h_{1}, h_{2}, r_{1}, r_{2}\right)
\end{gathered}
$$

and where $\beta\left(\theta ; h_{1}, h_{2}, r_{1}, r_{2}\right)$ was defined in Lemma 4.6.

Similar to the case $S_{3}(x)$, we have

$$
\begin{aligned}
& T^{2 l} \sum_{11} \frac{1}{h_{1}^{1 / 4} h_{2}^{1 / 4} r_{1}^{5 / 4} r_{2}^{5 / 4}} \min \left(T^{1 / 2}, \frac{1}{\left|\alpha\left(\theta ; h_{1}, h_{2}, r_{1}, r_{2}\right)\right|}\right) \\
\ll & T^{2 l} \sum_{11} \frac{1}{h_{1}^{1 / 4} h_{2}^{1 / 4} r_{1}^{5 / 4} r_{2}^{5 / 4}} \times \frac{1}{\left|\alpha\left(\theta ; h_{1}, h_{2}, r_{1}, r_{2}\right)\right|} \\
\ll & T^{2 l} \sum_{11} \frac{1}{h_{1}^{1 / 2} h_{2}^{1 / 2} r_{1}^{3 / 2} r_{2}^{3 / 2}} \ll T^{2 l} \mathcal{L}^{2} .
\end{aligned}
$$

By Lemma 4.6 we have $\left|\alpha\left(\theta ; h, h, r_{1}, r_{2}\right)\right| \gg h^{1 / 2}\left(r_{1} r_{2}\right)^{-1 / 4}$ under the condition 
$S C\left(\Sigma_{12}\right)$. Thus

$$
\begin{aligned}
& T^{2 l} \sum_{12} \frac{1}{h^{1 / 2} r_{1}^{5 / 4} r_{2}^{5 / 4}} \min \left(T^{1 / 2}, \frac{1}{\left|\alpha\left(\theta ; h, h, r_{1}, r_{2}\right)\right|}\right) \\
\ll & T^{2 l} \sum_{12} \frac{1}{h^{1 / 2} r_{1}^{5 / 4} r_{2}^{5 / 4}} \times \frac{1}{\left|\alpha\left(\theta ; h, h, r_{1}, r_{2}\right)\right|} \\
\ll & T^{2 l} \sum_{h, r_{1}, r_{2}} \frac{1}{h r_{1} r_{2}} \ll T^{2 l} \mathcal{L}^{3} .
\end{aligned}
$$

Now we estimate the sum $\Sigma_{13}$. By a splitting argument we have

$$
\begin{aligned}
& \sum_{13} \frac{1}{h_{1}^{1 / 4} h_{2}^{1 / 4} r_{1}^{5 / 4} r_{2}^{5 / 4}} \min \left(T^{1 / 2}, \frac{1}{\left|\alpha\left(\theta ; h_{1}, h_{2}, r_{1}, r_{2}\right)\right|}\right) \\
\ll & \mathcal{L}^{4} \sum_{14} \frac{1}{h_{1}^{1 / 4} h_{2}^{1 / 4} r_{1}^{5 / 4} r_{2}^{5 / 4}} \min \left(T^{1 / 2}, \frac{1}{\left|\alpha\left(\theta ; h_{1}, h_{2}, r_{1}, r_{2}\right)\right|}\right) \\
\ll & \left(H_{1} H_{2}\right)^{-1 / 4}\left(N_{1} N_{2}\right)^{-5 / 4} \mathcal{L}^{4} \sum_{14} \min \left(T^{1 / 2}, \frac{1}{\left|\alpha\left(\theta ; h_{1}, h_{2}, r_{1}, r_{2}\right)\right|}\right) \\
\ll & U_{1}+U_{2}
\end{aligned}
$$

for some $\left(H_{1}, H_{2}, N_{1}, N_{2}\right)$ for which

$$
1 \ll H_{j} \ll H, H_{j} \ll N_{j} \ll H 2^{2 J}(j=1,2),
$$

where

$$
\begin{aligned}
& U_{1}=T^{1 / 2}\left(H_{1} H_{2}\right)^{-1 / 4}\left(N_{1} N_{2}\right)^{-5 / 4} \mathcal{L}^{4} \times \mathcal{A}_{\theta}\left(H_{1}, H_{2}, N_{1}, N_{2} ; T^{-1 / 2}\right), \\
& U_{2}=\left(H_{1} H_{2}\right)^{-1 / 4}\left(N_{1} N_{2}\right)^{-5 / 4} \mathcal{L}^{4} \sum_{15} \frac{1}{\left|\alpha\left(\theta ; h_{1}, h_{2}, r_{1}, r_{2}\right)\right|}, \\
& S C\left(\Sigma_{14}\right): h_{j} \sim H_{j}, r_{j} \sim N_{j}(j=1,2), h_{1} \neq h_{2}, \\
& 0<\left|\alpha\left(\theta ; h_{1}, h_{2}, r_{1}, r_{2}\right)\right|<\frac{1}{10} \beta^{1 / 4}\left(\theta ; h_{1}, h_{2}, r_{1}, r_{2}\right), \\
& S C\left(\Sigma_{15}\right): h_{j} \sim H_{j}, r_{j} \sim N_{j}(j=1,2), h_{1} \neq h_{2}, \\
& T^{-1 / 2}<\left|\alpha\left(\theta ; h_{1}, h_{2}, r_{1}, r_{2}\right)\right|<\frac{1}{10} \beta^{1 / 4}\left(\theta ; h_{1}, h_{2}, r_{1}, r_{2}\right) .
\end{aligned}
$$

We first estimate $U_{1}$. By Lemma 4.4 we have

$$
\begin{aligned}
U_{1} & \ll T^{1 / 2}\left(H_{1} H_{2}\right)^{-1 / 4}\left(N_{1} N_{2}\right)^{-5 / 4} \mathcal{L}^{4}\left(T^{-1 / 2}\left(H_{1} H_{2} N_{1} N_{2}\right)^{3 / 4}+\left(H_{1} H_{2} N_{1} N_{2}\right)^{1 / 2} \mathcal{L}^{2}\right) \\
& \ll\left(H_{1} H_{2}\right)^{1 / 2}\left(N_{1} N_{2}\right)^{-1 / 2} \mathcal{L}^{4}+T^{1 / 2}\left(H_{1} H_{2}\right)^{1 / 4}\left(N_{1} N_{2}\right)^{-3 / 4} \mathcal{L}^{6} \\
& \ll \mathcal{L}^{4}+T^{1 / 2}\left(N_{1} N_{2}\right)^{-1 / 2} \mathcal{L}^{6}
\end{aligned}
$$


if noting that $N_{j} \gg H_{j}(j=1,2)$. Suppose $\left(h_{1}, h_{2}, r_{1}, r_{2}\right)$ satisfies the conditions of $\Sigma_{14}$. By Lemma 4.6 we have

$$
\left|\alpha\left(\theta ; h_{1}, h_{2}, r_{1}, r_{2}\right)\right| \gg\left(H_{1} H_{2}\right)^{-\frac{1}{4}-\frac{\gamma}{2}-\frac{\varepsilon}{2}}\left(N_{1} N_{2}\right)^{-\frac{1}{4}}
$$

which combining $\left|\alpha\left(\theta ; h_{1}, h_{2}, r_{1}, r_{2}\right)\right| \leq T^{-1 / 2}$ gives

$$
\left(H_{1} H_{2}\right)^{\frac{\gamma}{2}+\frac{1}{4}+\frac{\varepsilon}{2}}\left(N_{1} N_{2}\right)^{\frac{1}{4}} \gg T^{1 / 2} .
$$

Hence

$$
\left(N_{1} N_{2}\right)^{\frac{1}{2}+\frac{\gamma}{2}+\frac{\varepsilon}{2}} \gg\left(H_{1} H_{2}\right)^{\frac{\gamma}{2}+\frac{1}{4}+\frac{\varepsilon}{2}}\left(N_{1} N_{2}\right)^{\frac{1}{4}} \gg T^{1 / 2}
$$

namely

$$
N_{1} N_{2} \gg T^{\frac{1}{1+\gamma+\varepsilon}}
$$

From the above estimates we get

$$
U_{1} \ll T^{1 / 2-1 / 2(1+\gamma)+\varepsilon} .
$$

Now we estimate $U_{2}$. By a splitting argument we have

$$
U_{2} \ll \eta^{-1}\left(H_{1} H_{2}\right)^{-1 / 4}\left(N_{1} N_{2}\right)^{-5 / 4} \mathcal{L}^{5} \times \mathcal{A}_{\theta}\left(H_{1}, H_{2}, N_{1}, N_{2} ; \eta\right)
$$

for some $T^{-1 / 2} \ll \eta \ll\left(H_{1} H_{2} N_{1} N_{2}\right)^{1 / 4}$. By Lemma 4.6 we get

$$
\begin{aligned}
U_{2} & \ll \eta^{-1}\left(H_{1} H_{2}\right)^{-1 / 4}\left(N_{1} N_{2}\right)^{-5 / 4} \mathcal{L}^{5}\left(\eta\left(H_{1} H_{2} N_{1} N_{2}\right)^{3 / 4}+\left(H_{1} H_{2} N_{1} N_{2}\right)^{1 / 2} \mathcal{L}^{2}\right) \\
& \ll\left(H_{1} H_{2}\right)^{1 / 2}\left(N_{1} N_{2}\right)^{-1 / 2} \mathcal{L}^{5}+\eta^{-1}\left(H_{1} H_{2}\right)^{1 / 4}\left(N_{1} N_{2}\right)^{-3 / 4} \mathcal{L}^{7} \\
& \ll \mathcal{L}^{5}+\eta^{-1}\left(H_{1} H_{2}\right)^{1 / 4}\left(N_{1} N_{2}\right)^{-3 / 4} \mathcal{L}^{7}
\end{aligned}
$$

From Lemma 4.6 we get

$$
\eta \gg\left(H_{1} H_{2}\right)^{-\frac{1}{4}-\frac{\gamma}{2}-\frac{\varepsilon}{2}}\left(N_{1} N_{2}\right)^{-\frac{1}{4}},
$$

which combining $\eta \gg T^{-1 / 2}$ gives

$$
\eta^{-1} \ll \min \left(T^{1 / 2},\left(H_{1} H_{2}\right)^{\frac{\gamma}{2}+\frac{1}{4}+\frac{\varepsilon}{2}}\left(N_{1} N_{2}\right)^{\frac{1}{4}}\right) .
$$

Thus we have

$$
\begin{aligned}
(5.21) U_{2} \ll & \mathcal{L}^{5}+\min \left(T^{1 / 2},\left(H_{1} H_{2}\right)^{\frac{\gamma}{2}+\frac{1}{4}+\frac{\varepsilon}{2}}\left(N_{1} N_{2}\right)^{\frac{1}{4}}\right) \\
& \times\left(H_{1} H_{2}\right)^{1 / 4}\left(N_{1} N_{2}\right)^{-3 / 4} \mathcal{L}^{7} \\
\ll & \mathcal{L}^{5}+\min \left(T^{1 / 2}\left(H_{1} H_{2}\right)^{1 / 4}\left(N_{1} N_{2}\right)^{-3 / 4},\left(H_{1} H_{2}\right)^{\frac{1+\gamma}{2}+\frac{\varepsilon}{2}}\left(N_{1} N_{2}\right)^{-\frac{1}{2}}\right) \\
\ll & \mathcal{L}^{5}+\min \left(T^{1 / 2}\left(H_{1} H_{2}\right)^{-1 / 2},\left(H_{1} H_{2}\right)^{\frac{\gamma}{2}+\frac{\varepsilon}{2}}\right) \\
\ll & \mathcal{L}^{5}+\left(T^{1 / 2}\left(H_{1} H_{2}\right)^{-1 / 2}\right)^{\gamma /(1+\gamma)}\left(\left(H_{1} H_{2}\right)^{\frac{\gamma}{2}+\frac{\varepsilon}{2}}\right)^{1 /(1+\gamma)} \\
\ll & T^{1 / 2-1 / 2(1+\gamma)+\varepsilon} .
\end{aligned}
$$


From (5.16)-(5.21) we get

$$
\int_{T}^{2 T} S_{2}(x) d x \ll T^{2 l+1 / 2-1 / 2(1+\gamma)+\varepsilon},
$$

which combining (5.11), (5.14) and (5.15) implies that

$$
\int_{T}^{2 T} R_{11}^{2}(x, H) d x=\frac{2^{3-2 l} C_{l, \theta}}{\pi^{2}(l-1) !^{2}} \int_{T}^{2 T} x^{2 l-\frac{1}{2}} d x+O\left(T^{2 l+1 / 2-1 / 2(1+\gamma)+\varepsilon}\right)
$$

\subsubsection{Mean square of $R_{1}(x, H)$}

We have

$$
R_{1}^{2}(x, H)=R_{11}^{2}(x, H)+O\left(\left|R_{11}(x, H)\right| T^{l-1 / 2+\frac{\gamma}{4 \gamma+2}+\varepsilon}+T^{2 l-1+\frac{2 \gamma}{4 \gamma+2}+2 \varepsilon}\right) .
$$

By (5.7) , (5.23) and Cauchy's inequality we get

$$
T^{l-1 / 2+\frac{\gamma}{4 \gamma+2}} \int_{T}^{2 T}\left|R_{11}(x, H)\right| d x \ll T^{2 l+\frac{4 \gamma+1}{8 \gamma+4}+\varepsilon} .
$$

From (5.23), (5.24) and (5.25) we get

$$
\int_{T}^{2 T} R_{1}^{2}(x, H) d x=\frac{2^{3-2 l} C_{l, \theta}}{\pi^{2}(l-1) !^{2}} \int_{T}^{2 T} x^{2 l-\frac{1}{2}} d x+O\left(T^{2 l+\frac{4 \gamma+1}{8 \gamma+4}+\varepsilon}\right) .
$$

\subsection{Mean square of $R_{2}(x, H)$}

We first study the integral $\int_{T}^{2 T} G(x, H) d x$. We have

$$
\begin{aligned}
\int_{T}^{2 T} G(x, H) d x & \ll \int_{T}^{2 T} \sum_{m \leq \sqrt{\frac{2 T}{\theta}}} \min \left(1, \frac{1}{H\left\|\frac{x}{2 m}-\frac{\theta m}{2}+\frac{l}{2}\right\|}\right) d x \\
& \ll \sum_{m \leq \sqrt{\frac{2 T}{\theta}}} \int_{T}^{2 T} \min \left(1, \frac{1}{H\left\|\frac{x}{2 m}-\frac{\theta m}{2}+\frac{l}{2}\right\|}\right) d x \\
& \ll \sum_{m \leq \sqrt{\frac{2 T}{\theta}}} m \int_{\frac{T}{2 m}-\frac{\theta m}{2}+\frac{l}{2}}^{\frac{T}{m}-\frac{\theta m}{2}+\frac{l}{2}} \min \left(1, \frac{1}{H\|u\|}\right) d u \\
& \ll \sum_{m \leq \sqrt{\frac{2 T}{\theta}}} T \int_{0}^{1 / 2} \min \left(1, \frac{1}{H\|u\|}\right) d u \\
& \ll T^{3 / 2} H^{-1} \log H \ll T^{-1 / 2} \mathcal{L} .
\end{aligned}
$$


So we have(noting trivially $G(x, H) \ll T^{1 / 2}$ )

$$
\begin{aligned}
\int_{T}^{2 T} R_{2}^{2}(x, H) d x & \ll T^{2 l}+T^{2 l-1} \int_{T}^{2 T} G^{2}(x, H) d x \\
& \ll T^{2 l}+T^{2 l-1 / 2} \int_{T}^{2 T} G(x, H) d x \\
& \ll T^{2 l}+T^{2 l-1} \mathcal{L} \ll T^{2 l} .
\end{aligned}
$$

\subsection{Proof of Theorem 1}

We have

$$
R^{2}(2 \pi x)=R_{1}^{2}(x, H)+2 R_{1}(x, H) R_{2}(x, H)+R_{2}^{2}(x, H) .
$$

From (5.26), (5.28) and Cauchy's inequality we have

$$
\int_{T}^{2 T} R_{1}(x, H) R_{2}(x, H) d x \ll T^{2 l+1 / 4} .
$$

From (5.26), (5.28), (5.29) and (5.30) we get

$$
\int_{T}^{2 T} R^{2}(2 \pi x) d x=\frac{2^{3-2 l} C_{l, \theta}}{\pi^{2}(l-1) !^{2}} \int_{T}^{2 T} x^{2 l-\frac{1}{2}} d x+O\left(T^{2 l+\frac{4 \gamma+1}{8 \gamma+4}+\varepsilon}\right) .
$$

Hence

$$
\begin{aligned}
\int_{1}^{T} R^{2}(2 \pi x) d x & =\frac{2^{3-2 l} C_{l, \theta}}{\pi^{2}(l-1) !^{2}} \int_{1}^{T} x^{2 l-\frac{1}{2}} d x+O\left(T^{2 l+\frac{4 \gamma+1}{8 \gamma+4}+\varepsilon}\right) \\
& =\frac{2^{3-2 l} C_{l, \theta}}{(2 l+1 / 2) \pi^{2}(l-1) !^{2}} T^{2 l+1 / 2}+O\left(T^{2 l+\frac{4 \gamma+1}{8 \gamma+4}+\varepsilon}\right) .
\end{aligned}
$$

Now Theorem 1 follows from (5.32).

\section{References}

[1] V. Bentkus, F. Götze, Lattice point problems and distribution of values of quadratic forms, Ann. of Math. (2)50: 3(1999), 977-1027.

[2] P. H. Bérard, On the wave equation on a compact Riemannian manifold without conjugate points, Math. Z. 155:3(1977), 249-276. 
[3] L. Bleher, On the distribution of the number of lattice points inside a family of convex ovals, Duke Math. J. 67: 3(1992), 461-481.

[4] D. Chung, Y. N. Petridis and J. Toth, The remainder in Weyl's law for Heisenberg manifolds II, Bonner Mathematische Schriften, Nr. 360, Bonn, 2003, 16 pages.

[5] G. B. Folland, Harmonic Analysis in Phase Space, Princeton University Press(1989), 9-73.

[6] F. Fricker, Einführung in die Gitterpunketlehre, [Introduction to lattice point theory] Lehrbücher und Monographien aus dem Gebiete der Exakten Wissenschaften(LMW), Mathematische Reihe[Textbooks and Monographs in the Exact Sciences]73, Birkhäuser Verlag, Basel-Boston, Mas., 1982.

[7] C. Gordon, E. Wilson, The spectrum of the Laplacian on Riemannian Heisenberg manifolds, Michigan Math. J. 33(2) (1986), 253-271.

[8] F. Götze, Lattice point problems and values of quadratic forms, Inventiones Mathematicae157(2004), 195-226.

[9] G. H. Hardy, On the expression of a number as the sum of two squares, Quart. J Math. 46(1915), 263-283.

[10] D. R. Heath-Brown, The Piatetski-Shapiro prime theorem, J.of Number theory, Vol16(1983), 242-266.

[11] L. Hörmander, The spectral function of an elliptic operator, Acta Math.121(1968), 193-218.

[12] M. N. Huxley, Exponential sums and lattice points III, Proc. London Math. Soc. 87 (3) (2003), 591-609.

[13] V.YA. Ivrii, Precise Spectral Asymptotics for elliptic Operators Acting in Fibrings over Manifolds with Boundary, Springer Lecture notes in Mathematics 1100(1984).

[14] I. Kátai, The number of lattice points in a circle (in Russian), Ann. Univ. Sci. Budapest Rolando Eötvös, Sect. Math., 8 (1965), 39-60.

[15] M. Khosravi, Third moment of the remainder error term in Weyl's law for Heisenberg manifolds, arXiv: 0711.0073..

[16] M. Khosravi, Y. Petridis, The remainder in Weyl's law for $n$-dimensional Heisenberg manifolds, Proc. of the American Math. Soc. 133(2005), 3561-3571. 
[17] M. Khosravi, J. Toth, Cramér's formula for Heisenberg manifolds, Ann. de l'institut Fourier 55(2005), 2489-2520.

[18] A. Y. Khinchin, Zur metrischen Theorie der diophantischen Approximationen, Math. Z. 24 (1926), no. 4, 706-714.

[19] M. Kuhleitner, W. G. Nowak, The asymptotic behaviour of the mean-square of fractional part sums, Proc. Edinb. Math. Soc. 43(2000), 309-323.

[20] S. H. Min, The methods of number theory(in Chinese), Science Press, Beijing: 1981.

[21] Y. Petribis, J. Toth, The remainder in Weyl's law for Heisenberg manifolds, J. Differential Geom. 60(2002), 455-483.

[22] K. F. Roth, Rational aproximation to algebraic numbers, Mathematica 2(1955), $1-20$.

[23] E. M. Stein, Harmonic Analysis, Princeston University Press(1993), 527-574.

[24] Kai-Man Tsang, Higher-power moments of $\Delta(x), E(t)$ and $P(x)$, Proc. London Math. Soc.(3)65(1992), 65-84.

[25] J. D. Vaaler, Some extremal functions in Fourier analysis, Bull. Amer. Math. Soc. 12 (1985), 183-216.

[26] I. M. Vinogradov, Special variants of the method of trigonometric sums, (Nauka, Moscow), 1976; English transl. in his Selected works (Springer-Verlag), 1985.

[27] A. V. Volovoy, Improved two-term asymptotics for the eigenvalue distribution function of an elliptic operator on a compact manifold, Comm. Partial Differemtial Equations15: 11(1990), 1509-1563.

[28] Wenguang Zhai, On higher-power moments of $\Delta(x)$ (II), Acta Arith. 114 (2004), 35-54.

[29] Wenguang Zhai, On higher-power moments of $\Delta(x)$ (III), Acta Arith. 118 (2005), 263-281.

[30] Wenguang Zhai, On the error term in Weyl's law for the Heisenberg manifolds, Acta Arith., in press(see also arXiv: 0805.3856). 
School of Mathematical Sciences, Shandong Normal University,

Jinan, Shandong, 250014,

P.R.China

E-mail:zhaiwg@hotmail.com

Current address:

Department of Mathematics,

China University of Mining and Technology(Beijing),

Beijing 100083, P. R. China 